ARTICLE

\title{
Paper microfluidic implementation of loop mediated isothermal amplification for early diagnosis of hepatitis $\mathrm{C}$ virus
}

\author{
Weronika Witkowska McConnell (1) 1,2, Chris Davis', Suleman R. Sabir', Alice Garrett²,
}

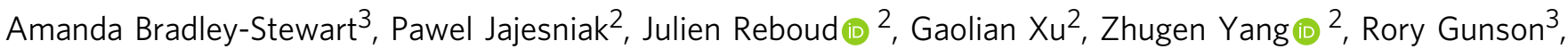
Emma C. Thomson (i) ${ }^{1 凶} \&$ Jonathan M. Cooper (i) ${ }^{2 凶}$

The early diagnosis of active hepatitis $\mathrm{C}$ virus (HCV) infection remains a significant barrier to the treatment of the disease and to preventing the associated significant morbidity and mortality seen, worldwide. Current testing is delayed due to the high cost, long turnaround times and high expertise needed in centralised diagnostic laboratories. Here we demonstrate a user-friendly, low-cost pan-genotypic assay, based upon reverse transcriptase loop mediated isothermal amplification (RT-LAMP). We developed a prototype device for point-of-care use, comprising a LAMP amplification chamber and lateral flow nucleic acid detection strips, giving a visually-read, user-friendly result in $<40 \mathrm{~min}$. The developed assay fulfils the current guidelines recommended by World Health Organisation and is manufactured at minimal cost using simple, portable equipment. Further development of the diagnostic test will facilitate linkage between disease diagnosis and treatment, greatly improving patient care pathways and reducing loss to follow-up, so assisting in the global elimination strategy.

\footnotetext{
${ }^{1}$ MRC-University of Glasgow, Centre for Virus Research, University of Glasgow, Glasgow, UK. ${ }^{2}$ Division of Biomedical Engineering, James Watt School of Engineering, University of Glasgow, Glasgow, UK. ${ }^{3}$ West of Scotland Specialist Virology Centre, New Lister Building, Glasgow Royal Infirmary, Glasgow, UK.

凶email: emma.thomson@glasgow.ac.uk; jon.cooper@glasgow.ac.uk
} 
epatitis $\mathrm{C}$ virus (HCV) is a major cause of liver-related mortality with 71 million people chronically infected globally. Recent advances in direct acting antiviral treatments have improved cure rates to $>95 \%$. However, currently an estimated $80 \%$ of all infected individuals are unaware of their status due to the asymptomatic nature of infection. Many of these patients will remain undiagnosed until irreversible clinical manifestations, such as liver cirrhosis and hepatocellular carcinoma, develop, contributing to the $400,000 \mathrm{HCV}$ related deaths reported every year ${ }^{1}$.

The World Health Organisation (WHO) has established a global elimination strategy to reach significant disease reduction targets by $2030^{2,3}$. The most challenging obstacle in achieving this ambitious goal is the rapid diagnosis of patients and their integration into an appropriate clinical care pathway for treatment ${ }^{3}$.

Current diagnostic strategies rely on testing for anti-HCV antibodies followed by RNA or core antigen detection ${ }^{4}$. This twostep process requires a centralised laboratory infrastructure and delays active $\mathrm{HCV}$ infection diagnosis by significant periods of time. Successful implementation is also limited by cost, long turnaround times and the high level of expertise required for diagnostic testing. High risk groups, including people-who-injectdrugs (PWID), are often lost to follow-up due to the multiple visits required for HCV diagnosis. Additionally, absent or delayed seroconversion, particularly in immunocompromised patients, may reduce overall rates of diagnosis ${ }^{5}$.

Most individuals with $\mathrm{HCV}$ reside in low and middle-income countries (LMICs) with only limited access to diagnosis ${ }^{1,6}$. Although recent advances in HCV nucleic acid amplification tests (NAATs) have provided a possible solution for improving the management of HCV diagnosis, the existing diagnostic platforms still pose several limitations, including the high cost and training requirements associated with polymerase chain reaction (PCR) assays, especially when used in decentralised testing ${ }^{7,8}$. Not only are simpler and cheaper NAATs for point-of-care (POC) testing required in order to improve $\mathrm{HCV}$ diagnosis and eliminate the need for follow-up visits 3 , but importantly, the selection of a universal NAATs' target is needed. HCV, like many RNA viruses, exhibits high genetic diversity, with eight distinct HCV genotypes and at least 90 different subtypes identified with varied prevalence gobally 9,10 .

Loop mediated isothermal amplification (LAMP) assays provide high sensitivity and specificity through the use of 4-6 primers, which target 6-8 regions within a sequence of interest $t^{11,12}$. The amplification reaction takes place at a constant temperature between $60-65^{\circ} \mathrm{C}$, offering a cheaper alternative to the traditional PCR assays, with minimal equipment requirements, which has resulted in its use in decentralised (POC) settings to detect the presence of a variety of pathogens ${ }^{13-15}$.

Recently, LAMP assays have also been developed for HCV detection in centralised facilities. Colorimetric outputs have also been developed to enable the detection without bulky optical instrumentation with excellent performance for some genotypes (e.g. Hongjaisee S. et al. ${ }^{16}$ showed $100 \%$ sensitivity with genotype 6). However, these assays showed limited diagnostic performance when used with varied genotypes or low viral loads ${ }^{17-19}$. Their application as POC tests is thus restricted, especially in $\mathrm{LMICs}^{20}$, where a wide variation in the genetic diversity may lead to a lower efficacy of tests.

In this study, we now demonstrate a pan-genotypic $\mathrm{HCV}$ LAMP assay, which we validated in a prototype POC diagnostic device as a paper-microfluidic, visually read lateral flow test. The assay was validated as part of a double-blind clinical study of samples from patients with a range of viral loads and genotypes as determined by a highly sensitive gold-standard Abbott RealTime RT-PCR HCV test, within a clinical reference laboratory and our own in-house HCV RT- PCR. We subsequently implemented the LAMP nucleic acid detection strip method into a portable and user-friendly device, with an easy-to-use readout for target detection.

\section{Results}

Optimisation of HCV LAMP primers. Previously published LAMP primers were selected based on evidence of a low limit of detection from HCV RNA $(50 \mathrm{IU} / \mathrm{mL})^{17}$. In this study, the use of an additional accelerating primer (AP, Table 1) ensured improved sensitivity and specificity across several HCV genotypes. We analysed an alignment file of over 200 sequences of major HCV genotypes and subtypes ${ }^{21}$. The majority of the observed mismatches were in the middle of the primers (Fig. S1). We only noted one mismatch within the last base pair of the $3^{\prime}$ end of the backward loop primer (BLP), within genotype 3 sequences. Focussing on genotype 3 and in order to further improve the previously published assay, we removed the cytosine mismatch at the $3^{\prime}$ end of BLP. Additionally, a cytosine was added at the beginning of the primer, in order to conserve the primer melting temperature (Fig. S1 and Table 1). The removal of the cytosine mismatch and the subsequent use of the new primer improved the time to positivity by $21 \%$ in genotype 3 (Fig. S2). As a confirmation, the new BLP was also compared to the original BLP performance on genotype 1 targets, which, did not result in significant differences in the assay performance as there were no mismatches within this genotype.

Clinical sensitivity and specificity. Using optimised primers, we conducted a double-blind study of LAMP on RNA and cDNA from samples with a range of viral loads and from different HCV genotypes, including a recently identified genotype 7 a (Table 2$)^{22}$.

The performance of HCV LAMP using RNA samples (RTLAMP) directly and HCV LAMP using CDNA as template were compared with a highly sensitive in-house HCV RT- PCR by genotype and viral load (Tables 2 and 3). The in-house RT-PCR assay has been characterised previously as a quantitative assay $\left(\mathrm{qRT}-\mathrm{PCR}^{23}\right)$.

HCV RT-LAMP and HCV LAMP assays detected 96 and 97 HCV samples out of 100 respectively, comparable with the RTPCR assay which detected 96/100 samples. The false negatives correlated well between the three assays (in two cases false negatives were due to low viral loads, $\leq 3.95 \log _{10} \mathrm{IU} / \mathrm{mL}$ ). RTLAMP failed to detect a single genotype 4 sample with high viral load $\left(5.64 \log _{10} \mathrm{IU} / \mathrm{mL}\right)$. RT-LAMP and LAMP assays both exhibited high specificity, correctly identifying 91 and 90 control

\section{Table 1 LAMP primer sequences.}

\begin{tabular}{ll} 
Primer name & Sequence (5' $\left.\rightarrow \mathbf{3}^{\prime}\right)$ \\
\hline F3 & ACT CCA CCA TGA ATC ACT C \\
B3 & ATC AGG CAG TAC CAC AAG G \\
FIP & AGG CTG YAC GAC ACT CAT AC-CTG \\
& TGA GGA ACT ACT GTC TTC \\
BIP & GGA TMA ACC CRC TCA ATG CC-TCG \\
& CRA CCC AAC RCT AC \\
FLP & GCC ATG GCT AGA CGC T \\
BLP & CGT GCC CCC GCR AGA[C] \\
AP & TTC CGC AGA CCA CTA TGG CTC T \\
FLP BIO & [BIO] GCC ATG GCT AGA CGC T \\
BLP FITC & [FITC] CGT GCC CCC GCR AGA \\
\hline Primer sequences were as published by Yang et al.17, with modifications marked in bold, \\
underlined. The bracket shows the original cytosine position. \\
FIP forward internal primer, BIP backward internal primer, FLP forward loop primer, BLP backward \\
loop primer, AP accelerating primer, Bio Biotin, FITC fluorescein isothiocyanate.
\end{tabular}


Table 2 Sample detection by genotype and viral load.

\begin{tabular}{lllll} 
Genotype & RT-LAMP & LAMP & RT-PCR & $\begin{array}{l}\text { Total samples } \\
\text { tested (\%) }\end{array}$ \\
\hline 1 & 25 & 25 & 25 & $26(26 \%)$ \\
2 & 14 & 14 & 14 & $14(14 \%)$ \\
3 & 22 & 22 & 21 & $23(23 \%)$ \\
4 & 21 & 22 & 22 & $22(22 \%)$ \\
5 & 3 & 3 & 3 & $3(3 \%)$ \\
6 & 1 & 1 & 1 & $1(1 \%)$ \\
7 & 1 & 1 & 1 & $1(1 \%)$ \\
Unknown & 9 & 9 & 9 & $10(10 \%)$ \\
Viral load & & & Total & 100 \\
$($ log 10 IU/mL) & & & & \\
$1.7-3.95$ & 3 & 3 & 2 & $5(5 \%)$ \\
$4.4-4.85$ & 12 & 12 & 12 & $12(12 \%)$ \\
$5.2-5.98$ & 36 & 37 & 37 & $37(37 \%)$ \\
$6.05-6.97$ & 37 & 37 & 37 & $37(37 \%)$ \\
Unknown & 8 & 8 & 8 & $9(9 \%)$ \\
& & & Total & 100 \\
\hline
\end{tabular}

Experiments were performed in duplicate and at least one positive replicate was interpreted as a positive result.

Table 3 Sensitivity and specificity of HCV assays.

\begin{tabular}{llll} 
Method & RT-LAMP & LAMP & RT-PCR \\
\hline True positive & 96 & 97 & 96 \\
False negative & 4 & 3 & 4 \\
Total (sensitivity) & $100(96 \%)$ & $100(97 \%)$ & $100(96 \%)$ \\
True negative & 91 & 90 & 100 \\
False positive & 9 & 10 & 0 \\
Total (specificity) & $100(91 \%)$ & $100(90 \%)$ & $100(100 \%)$ \\
\hline Abbott RealTime HCV assay (RT-PCR) was used as the gold standard. & \\
\hline
\end{tabular}

samples out of 100 respectively, although our in-house RT-PCR assay had a higher specificity with no false positives.

Time to detection. In order to evaluate the impact of genotype and viral load on the performance of both HCV RT-LAMP and LAMP assay, we defined samples as positive when the fluorescence signal was at least ten standard deviations above the mean baseline fluorescence of the positive control. The time to positive was then determined as described in Fig. S3. The majority of positive samples were detected within $30 \mathrm{~min}$ (Fig. 1a, b). For RTLAMP, genotype 3 and 4 detection took longer than genotype 1 and 2. Similarly, for cDNA, genotype 3 positivity occurred later than all other genotypes. There was an inverse relationship between time to detection and viral load and significant differences occurred between the $1.70-3.95 \log _{10} \mathrm{IU} / \mathrm{mL}$ group, $4.40-4.85 \log _{10} \mathrm{IU} / \mathrm{mL}$ group and the 6.05-6.97 $\log _{10} \mathrm{IU} / \mathrm{mL}$ group for both RNA and cDNA (Fig. 1c, d).

Since most samples were detected within the first $30 \mathrm{~min}$, we assessed if this would be a suitable detection time period. Receiver operating characteristic (ROC) curves for both LAMP and the RT-PCR were used to determine the efficacy by which the assays would distinguish between HCV-positive and control samples at different time points or cycles (Fig. 2, Fig. S4 and Tables S1-2). Both RT-LAMP and LAMP had areas under the curve of 0.97 (Fig. 2A, B), showing close statistical similarity to the RT-PCR assay (Fig. S4 and Table S1). The cut-off of $<29 \mathrm{~min}$ for RTLAMP was equivalent to $95.0 \%$ sensitivity (95\% CI: 88.7-98.4\%) and $94.0 \%$ specificity (95\% CI: $87.4 \%-97.8 \%$ ) (Fig. $2 \mathrm{C}$ and Table S2). LAMP had the same sensitivity at $<29.75 \mathrm{~min}$, but the specificity was slightly lower-92.0\% (95\% CI: 84.8-96.5\%) (Fig. 2D and Table S3). To achieve the specificity of $98 \%$, recommended by the WHO, $(98.0 \%, 95 \%$ CI of $93.0-99.8 \%)$, the cut-off value would be set to $<24.75 \mathrm{~min}$ for RT-LAMP and $<26.75 \mathrm{~min}$ for LAMP, resulting in a sensitivity of $90.0 \%$ (95\% CI of $82.4-95.1 \%$ ) and $92.0 \%$ (95\% CI of 84.8-96.5\%) for RT-LAMP and LAMP, respectively (Fig. 2C, D, Tables $\mathrm{S} 1-3)^{24}$.

Analytical sensitivity and end-point detection. Analytical sensitivity of HCV LAMP was assessed using serial dilutions of plasmids containing HCV sub-genomic replicon over $40 \mathrm{~min}$ by three different detection methods; nucleic acid detection strips, gel electrophoresis and changes in fluorescence over time. The concentration of HCV ranged between $2.6 \log _{10}$ copies/reaction and $4.1 \log _{10}$ copies/reaction. Figure 3 shows the mechanism of strip detection (Fig. 3a), the assembly of the lateral flow device (Fig. 3b), and the results from the analytical sensitivity experiments (Fig. 3c). The lower limit of detection was the same for each method, below $2.6 \log _{10}$ copies/reaction (broadly equivalent to a detection threshold of 398 copies/reaction, which is lower than the recommended $\leq 3000 \mathrm{IU} / \mathrm{mL}$ limit of detection by (WHO) ${ }^{2}$.

The nucleic acid detection strips used in this study offered an easy-to-interpret pregnancy test-like result with two bands for a positive reaction and one (control) band for negative. The gel electrophoresis revealed a characteristic smear with ladder-like banding pattern for all samples except the negative control. The size of the initial bands ( $274 \mathrm{bp}$ ) correlate with the region between F3/B3 representing the initial stem loop formation of the reaction. As the reaction proceeded, larger constructs were created, with random termination, generating a other bands with a smear-like pattern ${ }^{11,12,17}$. The results correlated with the nucleic acid detection strips. There was an inverse relationship between viral load and time to positivity and all positive reactions were statistically different from the negative control $(p<0.0001)$. The double-blind study revealed that optimal sensitivity and specificity occur at $<30 \mathrm{~min}$ with a lower limit of detection of $417 \mathrm{IU} / \mathrm{mL}$.

Nucleic acid detection strips are easy to visualise and interpret and are known to correlate well with other detection methods and were thus used as the basis for the development of a low-cost, microfluidic HCV test, which could be used either in bedside and/ or POC settings. An enclosed lateral flow device containing a LAMP reaction chamber, valves and detection strips was manufactured in-house using methods adapted from previous studies (shown in Fig. 3b and Fig. S5) ${ }^{15}$. The LAMP chamber was incubated in a heat block for $30 \mathrm{~min}$ at $65^{\circ} \mathrm{C}$ by inserting the device upright (thus ensuring that the lateral flow strips were held away from high temperatures, Fig. S6). At the end of the reaction, the amplicons, generated within the LAMP chamber were eluted onto the lateral flow strips by pressing a blister pack/finger pump containing running buffer. The amplicons, labelled with both FITC and biotin ligand binding sites, contacted the end of the lateral flow devices and then were carried along the paper strips by capillarity, where they interacted with conjugation pads. This prototype HCV test device offers a cheap and user-friendly detection method together with the high sensitivity and specificity of the LAMP reaction.

To characterise the performance of the lateral flow detection devices, we tested a further 40 patient samples (20 HCV-positive and 20 healthy controls) on this platform and compared the results to our in-house qPCR assay (see 'Methods' for details). Results show no false positives (20/20 negatives detected correctly, Supplementary Table S4). Three clinically positive samples did not provide any Ct value on $\mathrm{QPCR}$, indicative of either or both of low viral loads or degradation of the RNA from the additional 
a

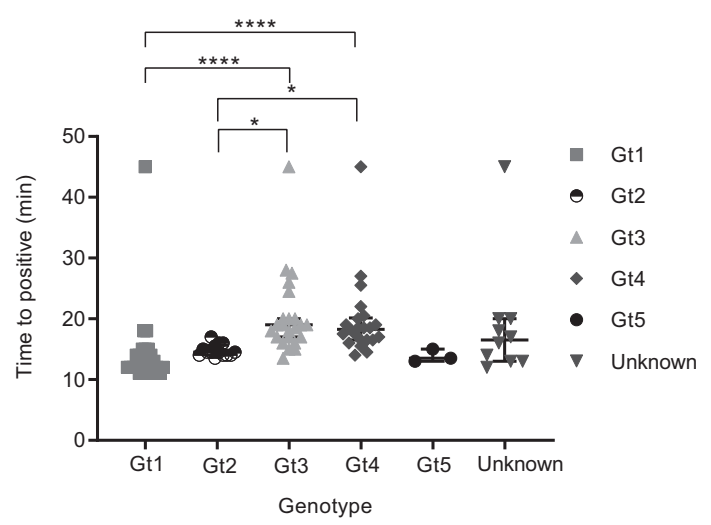

C

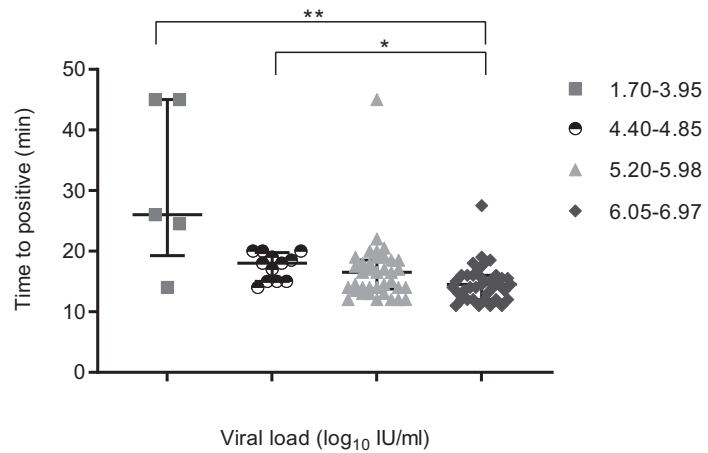

b

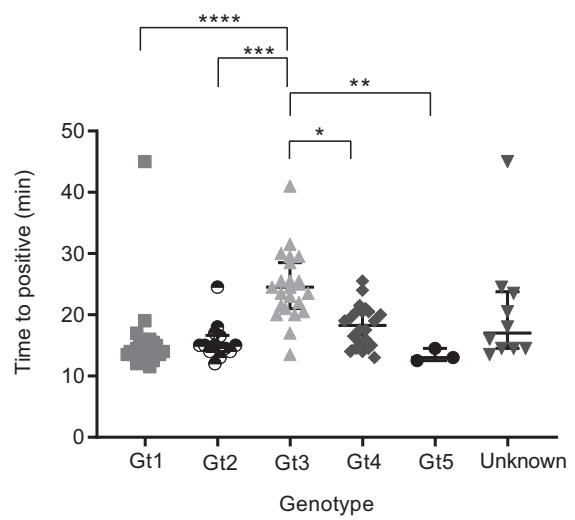

d

Fig. 1 The effect of genotype and viral load on detection. The central lines indicate median with interquartile range as error bars, and each point on the graph represents the mean of a sample run in duplicate. False negative samples were recorded as time to positive reaction at 45 min. Statistical analysis was performed using a one-sided non-parametric Kruskal-Wallis with Dunn's multiple comparisons test (one-sided). ${ }^{\star} p \leq 0.05,{ }^{\star \star} p \leq 0.01,{ }^{\star \star \star} p \leq 0.001$, ${ }^{\star \star \star \star} p \leq 0.0001$. a HCV RNA samples detection based on genotype (Gt): Gt1 (grey square, $n=26$ ), Gt2 (white and black disc, $n=14$ ), Gt3 (grey triangle, $n=23$ ), Gt4 (grey lozenge, $n=22$ ), Gt5 (black disc, $n=3$ ), Unknown (grey inverse triangle, $n=10$ ). Gt1 vs Gt3, $p<0.0001$, Gt1 vs Gt4, $p<0.0001$, Gt1 vs Unknown, $p=0.1524$, Gt2 vs Gt3, $p=0.0101$, Gt2 vs Gt4, $p=0.0284$, Gt3 vs Gt5, $p=0.1846$, Gt4 vs Gt5, $p=0.2814$. All remaining groups had $p$ values of $>0.9999$. b HCV cDNA samples detection based on genotype; Gt1 (grey square, $n=26$ ), Gt2 (white and black disc, $n=14$ ), Gt3 (grey triangle, $n=23$ ), Gt4 (grey lozenge, $n=22$ ), Gt5 (black disc, $n=3$ ), Unknown (grey inverse triangle, $n=10$ ). Gt1 vs Gt3, $p<0.0001, \mathrm{Gt} 1$ vs Gt4, $p=0.0562, \mathrm{Gt} 1$ vs Unknown, $p=0.3425$, Gt2 vs Gt3, $p=0.0002$, Gt3 vs Gt4, $p=0.0490, \mathrm{Gt} 3$ vs Gt5, $p=0.0035$, Gt3 vs Unknown, $p=0.3247, \mathrm{Gt} 4$ vs Gt5, $p=0.3701, \mathrm{Gt} 5$ vs Unknown, $p=0.5210$. All the remaining groups had $p$ values of $>0.9999$. c HCV RNA samples detection based on viral load: $1.7-3.95$ (grey square, $n=5$ ), 4.4-4.85 (black and white disc, $n=11$ ), 5.2-5.98 (grey triangle, $n=34$ ), 6.05-6.97 (grey lozenge, $n=44$ ). 1.7-3.95 vs 5.2-5.98, $p=0.1937$, $1.7-3.95$ vs $6.05-6.97, p=0.0095,4.4-4.85$ vs $6.05-6.97, p=0.0301,5.2-5.98$ vs 6.05-6.97, $p=0.4768,5.2-5.98$ vs Unknown, $p=0.4506,6.05-6.97$ vs Unknown, $p=0.0118$. All remaining groups had $p$ values of $>0.9999$. d HCV cDNA samples detection based on viral load; $1.7-3.95$ (grey square, $n=5$ ), 4.4-4.85 (black and white disc, $n=11$ ), 5.2-5.98 (grey triangle, $n=34$ ), 6.05-6.97 (grey lozenge, $n=44$ ). 1.7-3.95 vs 5.2-5.98, $p=0.0536,1.7-3.95$ vs 6.05-6.97, $p=0.0032,1.7-3.95$ vs Unknown, $p=0.5693,4.4-4.85$ vs 6.05-6.97, $p=0.0492,5.2-5.98$ vs 6.05-6.97, $p=0.9431,6.05-6.97$ vs Unknown, $p=0.7858$. All remaining groups had $p$ values of $>0.9999$. Source data are provided as a Source data file.

freeze-thaw cycles and longer storage. 14/17 positive samples were also identified correctly by the LAMP on lateral flow devices. Two of the false negative samples had $\mathrm{Ct}$ values above 30 for our in house qPCR, which is beyond our threshold for detection, indicating low viral loads. Consequently, only one sample $(\mathrm{Ct}$ 29) was negative for the lateral flow device, whilst being positive for qPCR, showing excellent agreement, in line with the results obtained for fluorescence read-outs and demonstrating the potential for this low-cost and user-friendly method.

\section{Discussion}

In this study, we aimed to develop a cheap, sensitive and specific bedside test for HCV. Using optimised primers, we validated its potential as a future POC/bedside test in a double-blind study of clinical samples with varied viral loads and comprising all major genotypes, including a recently identified genotype 7 sample 22 . Both RT-LAMP and LAMP performed well in this study, exceeding the standards set out by WHO for POC tests ${ }^{24}$. The samples were diagnosed by comparison with the gold standard Abbott RealTime HCV PCR assay within a validated clinical reference laboratory and further compared with an in-house RTPCR assay. A small number of samples that were not detected by RT-PCR were also missed by the LAMP assay, possibly due to low viral load.

The target product profile for diagnosis of $\mathrm{HCV}$ as recommended by WHO requires a minimum diagnostic specificity of $>98 \%$ and sensitivity of $>90-95 \%$ which was fulfilled by 
A

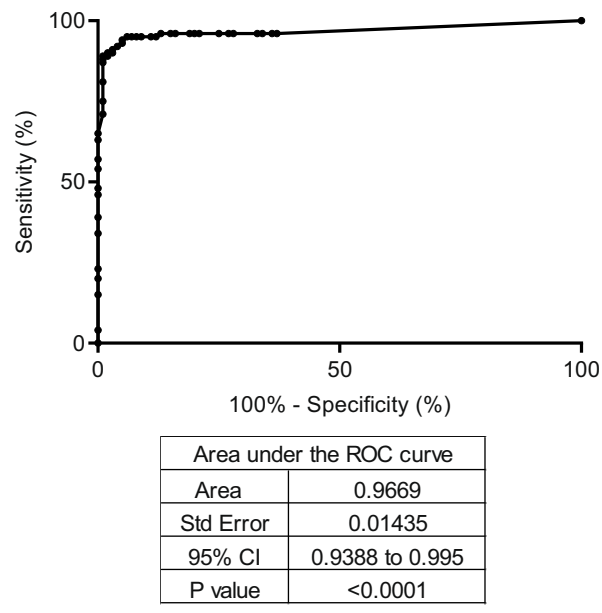

C

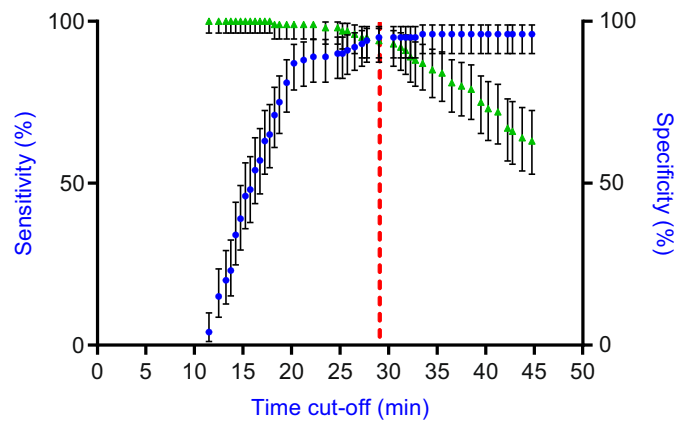

- Sensitivity $\Delta$ Specificity
B

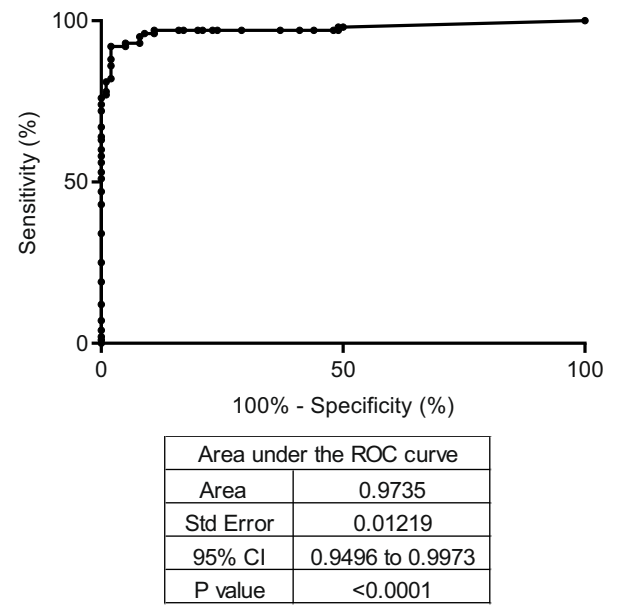

D

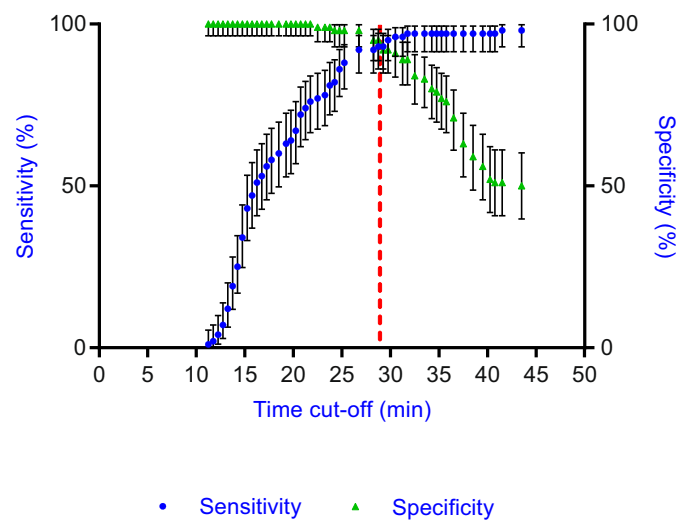

Fig. 2 ROC curves for LAMP assays. ROC curves were based on mean time to detection of $100 \mathrm{HCV}$ positive and $100 \mathrm{HCV}$ negative samples tested in a double-blind fashion. A, B ROC curve analysis, performed using the Clopper-Pearson Method (one-sided, no adjustments for multiple comparisons) for RTLAMP and RNA samples (a) and for LAMP and cDNA samples (B). C, D Graphs showing sensitivity (circle) and specificity (triangle) at different time points in minutes for RT-LAMP (C) and LAMP (D). Error bars are $95 \%$ confidence intervals. The dashed line indicates the cut-off time in minutes, where sensitivity and specificity is optimal. Source data are provided as a Source data file.

RT-LAMP at a cut-off of $<25 \mathrm{~min}^{22}$. However, the optimal analytical sensitivity of RT-LAMP assay, exceeding the WHO requirements, was reached at a cut-off of $<30 \mathrm{~min}$. The small number of false positive samples detected by LAMP versus RTPCR could represent a slightly increased risk of crosscontamination or the formation of primer-dimer structures detected by LAMP. Overall, false positives did not occur commonly in this study, as in other HCV LAMP studies ${ }^{17-19,25}$.

The analytical sensitivity of the assay at 40 min was $\sim 2.6 \log _{10}$ copies/reaction. In the original study from which we adapted our primers, the authors report a limit of detection of $50 \mathrm{IU} / \mathrm{mL}^{17}$. Both studies show that the assay falls well within the WHO's criteria for a point-of-care test $(3000 \mathrm{IU} / \mathrm{mL} \text { or below })^{2}$.

The current diagnostic algorithm for $\mathrm{HCV}$ relies on testing for anti-HCV antibodies and later for HCV RNA to confirm active infection $^{1}$. Although, rapid diagnostic tests for anti-HCV antibody detection have been developed and FDA approved, they are still limited by the window period in early HCV infection when antibodies are undetectable ${ }^{26}$. Detection of current infection has to be confirmed by highly sensitive and specific HCV RNA detection assays including RT-PCR and those are still primarily limited by the high costs involved and incur a significant delay for patient management, which can be associated with poor treatment initiation outcomes $^{27}$. The recent development of platforms such as GeneDrive and Cepheid Xpert, which are both CE-IVD certified, have improved the HCV diagnostic landscape with very high sensitivity and specificity, but require technical expertise and significant financial investment ${ }^{7,8}$. Both assays need a trained laboratory technician with at least one day of training to perform the assay. Furthermore, the cost of the GeneDrive platform is $\$ 5000$ and $\$ 30-40$ for each individual HCV test. In 
a

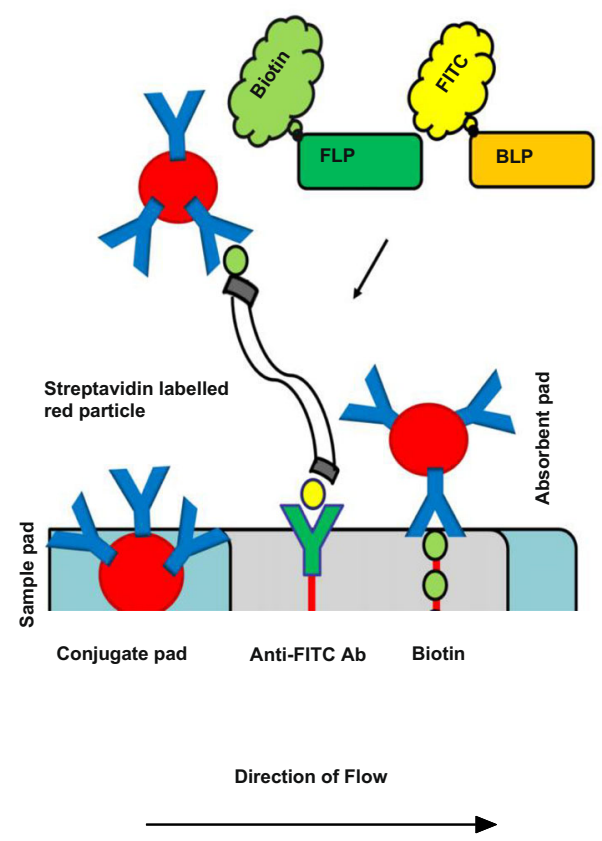

b
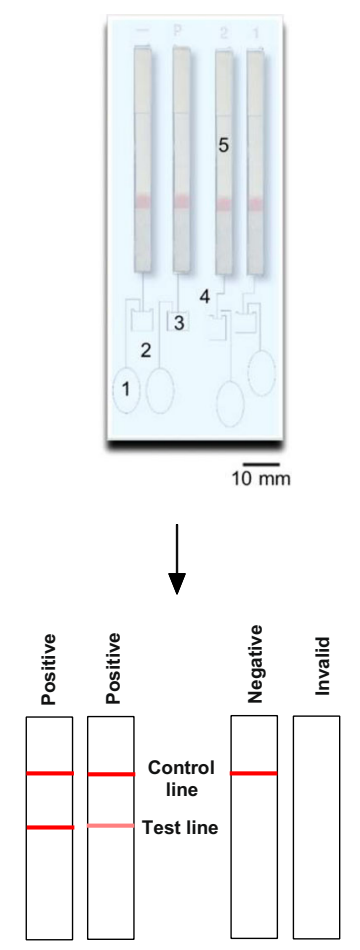

C

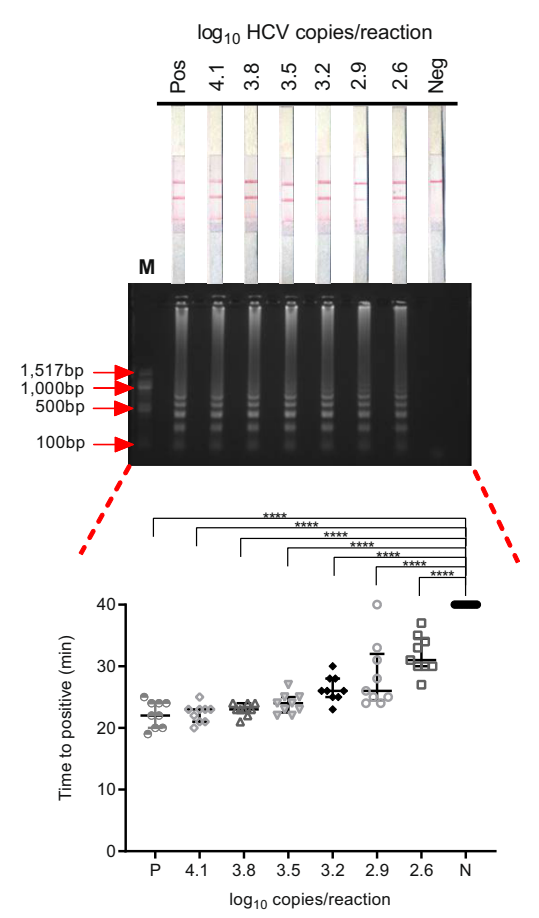

Fig. 3 Lateral flow detection of HCV LAMP assay. a The mechanism of the lateral flow strip ${ }^{15}$. Two primers, FLP and BLP, are pre-labelled with Biotin and FITC, respectively. The amplicon resulting from the LAMP reaction, contains both labelled primers as double-stranded DNA represented schematically by two gray lines. It is added onto the sample pad and moves towards the conjugate via capillary action. The streptavidin-labelled red particles bind with the Biotin (from the FLP primer) on the amplicon and together move towards the test line. The anti-FITC antibody (Ab) on the strip captures the amplicon via its FITC label (from the BLP primer) at the test line forming a band. Any unbound red particles move towards the control line where they are captured via the Biotin forming a second band. $\mathbf{b}$ The assembly and interpretation of the lateral flow devices. The device consists of the water chamber (1), connecting channels (2), four LAMP reaction chambers (3), channels (4) and lateral flow strips (5). Following the incubation period, two bands indicate a positive reaction, one band indicates a negative and no bands indicate invalid results. $\mathrm{P}$-positive, $\mathrm{N}$-negative, 1 and $2-$ sample in duplicate. c Analytical sensitivity of the lateral flow method (top panel) compared to gel electrophoresis (middle panel) and fluorescence over time (bottom panel). Serial dilutions of plasmid JFH1 replicon were made based on copy number per reaction $\left(\log _{10}\right)$. Black lines indicate median with interquartile range $(n=3$ biologically independent experiments, each with three technical replicates). The different symbols are for each dilution, to ease visualisation (grey and white disc-4.1, white lozenges-3.8, inverse triangles-3.5, black diamond-3.2, grey circles-2.9 and white squares $-2.6 \log _{10}$ copies/reaction, black disc is negative control-DI water). Statistical analysis for the fluorescence over time was performed using a parametric, one-way ANOVA. The F ratio $=55.56$ and the degrees of freedom $=65 .{ }^{\star \star \star \star} p \leq 0.0001$, Pos-positive HCV control, Neg-no template control, M-100 bp NEB DNA ladder, C-control line, T-test line. Source data are provided as a Source data file.

comparison, the authors of this study have previously estimated the cost of a similar LAMP assay to be $<\$ 10^{15}$.

The prototype lateral flow device developed in this study does not affect the sensitivity of the assay when compared to gel electrophoresis and fluorescence-based detection. Nucleic acid detection was carried out with an easy-to-interpret, pregnancy test-like visualisation output, requiring two labelled primers (Fluorescein isothiocyanate, FITC, labelled FLP and biotin, Bio, labelled BLP) specific to double-stranded HCV LAMP amplicons. Compared to our previous study ${ }^{15}$, which focussed on the detection of plasmodium DNA, we demonstrate this capability with the amplification of RNA for the detection of HCV. This contrasts with previous HCV LAMP studies that have used SYBR green for colorimetric detection with non-specific detection of double-stranded DNA, which may result in higher numbers of false positive results ${ }^{19}$.
In future work, we propose to integrate an RNA extraction system within the current lateral flow device. Candidate systems have already been described and have used LAMP directly from serum, urine and whole blood, for example in Zika virus detection, without significantly impairing assay performance ${ }^{28-30}$. Other studies have reported the use of paper microfluidics as a suitable RNA extraction method in conjugation with LAMP reactions including a recently published 30 -second nucleic acid extraction protocol ${ }^{31,32}$. The incorporation of such methods with our highly sensitive and specific HCV LAMP assay would allow for a sample-to-answer test result within $60 \mathrm{~min}$. A single visit would then be sufficient for diagnosis and initiation of treatment which could significantly improve the uptake of treatment in high risk groups including PWID and HIV infected men-who-havesex-with-men by reducing loss to follow-up ${ }^{33,34}$. PWID have a high global prevalence of HCV infection with an estimated 10 
million positive for anti-HCV antibodies in 148 countries $^{34,35}$. Several studies have demonstrated that POC testing is costeffective in high-risk groups as well as in countries with high HCV prevalence such as Egypt ${ }^{36,37}$. Since LAMP requires a stable temperature of $60-65^{\circ} \mathrm{C}$ with no cycling, reactions can be established with minimal equipment requirements, for example by using a commercially available thermos flask, water bath or coffee mug ${ }^{38}$. Using such methods, LAMP performs well in the field for the diagnosis of malaria ${ }^{15}$ and foot-and-mouth disease ${ }^{14}$ amongst others.

HCV LAMP is suitable for diagnosis across a range of different viral loads and genotypes. Differences were noted in the time to detection of genotype 3 with the LAMP assay and genotypes 3 and 4 for RT-LAMP, although these were significantly improved by the alteration of the BLP primer in optimisation experiments and were detected in $<30 \mathrm{~min}$. Assay performance could be optimised further by altering primer sequences based on the genotypes most prevalent in the area in which diagnosis is taking place.

In conclusion, we have developed a highly sensitive and specific HCV LAMP assay, which exceeds WHO requirements for a diagnostic test. Our prototype lateral flow device is a promising tool for user-friendly POC testing, eliminating the need for follow up visits between diagnosis and initiation of treatment. Future studies should evaluate prospective testing in the field in patients receiving $\mathrm{HCV}$ treatment.

\section{Methods}

Clinical samples and standards. 100 fully anonymised plasma samples from patients with chronic HCV infection were compared with $100 \mathrm{HCV}$-negative control samples. Samples, including healthy controls, were selected randomly from the West of Scotland Specialist Virology Centre (WoSSVC) via the Greater Glasgow and Clyde Health Bio-repository and the NHS Research Ethics Committee (REC), anonymised and processed from plasma at the MRC, Centre for Virus Research or from venous whole blood at the WoSSVC, Glasgow Royal Infirmary. Ethical approval was granted by the Greater Glasgow and Clyde Health Biorepository and the NHS Research Ethics Committee, application number 606. All samples were obtained with informed consent, with no compensation. Samples were assigned a numerical sample ID (1-200 or 1-40) randomly (using the random number generator in Microsoft Excel 365) and processed from venous whole blood at the WoSSVC. The correspondence (positive, negative and viral load when relevant) was held by the WoSSVC. The samples were provided blinded for processing.

The HCV viral load was quantified using the Abbott RealTime HCV assay. The viral load groupings were defined by the WoSSVC according to common clinical practice. Sensitivity and specificity testing was carried out using a double-blind study design. Analytical sensitivity was determined by serial two-fold dilutions of JFH1 subgenomic replicon containing the Guassia luciferase gene (pSGR-HCVJFH1-GLUC) ${ }^{39}$. The number of copies of replicon per reaction was calculated based on DNA mass and plasmid length on the NEB online calculator ${ }^{40}$.

40 samples stored at $-80^{\circ} \mathrm{C}$, from patients with HCV $(n=20)$ and negative controls $(n=20)$ were anonymised by WoSSVC staff. They were transported to the University of Glasgow on ice and stored at $-80^{\circ} \mathrm{C}$ until use. They were processed according to the procedure detailed below for the LAMP lateral flow devices and RT-PCR, in a double-blind fashion. The results were read independently by two assessors before unblinding.

RNA extraction and cDNA synthesis. RNA was extracted from $200 \mu \mathrm{L}$ of plasma using the Agencourt RNAdvance Blood Kit (Beckman Coulter) on the automated KingFisher ${ }^{\text {sa }}$ Flex Purification System, with a method adapted from the manufacturer's protocol. Samples were lysed with $300 \mu \mathrm{L}$ of Lysis buffer and $30 \mu \mathrm{L}$ of Proteinase K and the DNase stage was shortened to $5 \mathrm{~min}$. Purified RNA was eluted in $25 \mu \mathrm{L}$ of nuclease-free water. SuperScript III Reverse Transcriptase (Thermo Fisher Scientific) was used for complementary DNA synthesis from $11 \mu \mathrm{L}$ of eluted RNA with random hexamers as per the manufacturer's instructions.

HCV LAMP assay and product detection. LAMP reactions were run in a final volume of $25 \mu \mathrm{L}$, containing $15 \mu \mathrm{L}$ of ISO-001-RT Master Mix (Optigene, UK), $5 \mu \mathrm{L}$ of target DNA/RNA, $0.8 \mu \mathrm{M}$ of FIP and BIP, $0.4 \mu \mathrm{M}$ of FLP and BLP, $0.2 \mu \mathrm{M}$ of F3 and B3 primers and $0.4 \mu \mathrm{M}$ of AP. Primer sequences are shown in Table 1.

Reaction mixtures were incubated for $40-45 \mathrm{~min}$ at $65^{\circ} \mathrm{C}$. Real time monitoring of LAMP reactions was performed on a 7500 Fast Real-Time PCR (RT-PCR) machine (Applied Biosystems) using the SYBR green setting. For end-point detection, LAMP products were subjected to gel electrophoresis on a $1 \%$ agarose TAE gel dyed with ethidium bromide and the results detected under UV light. Additionally, LAMP products were visualised on nucleic acid detection strips (Ustar $^{\circledast}$, China). FLP and BLP were labelled with Biotin and FITC, respectively (Table 1), and the entire product was added to the strips and topped up with $\sim 100 \mu \mathrm{L}$ of nuclease free deionised water to enable the flow of the sample through the lateral strip using capillarity. The results were recorded within $10 \mathrm{~min}$ by the ESPON EXPRESSION 1680 Pro scanner with 300 dpi resolution. Images were processed in Microsoft PowerPoint 2016 software.

Lateral flow devices. Lateral flow devices containing chambers for LAMP reactions, finger pumps and nucleic acid detection strips were manufactured in-house on a laser cutter (Laserscript) from 2 mm-thick poly(methylmethacrylate) (Fig. 3b and Fig. S5a). Two single sided adhesive acetate films (MicroAmp Optical Adhesive Film; Thermo Scientific) were used to seal the devices and prevent evaporation during LAMP reactions. These devices are low-cost, estimated as follows: cartridge $<\$ 10 \mathrm{c}$, detection strip $\$ 1$, reagents (3 reactions (positive, negative, test)), $\$ 3$. Total $<\$ 5$.

HCV RT-PCR assay. An in-house RT-PCR with primer and probe sequences based on the $5^{\prime}$ UTR (JFH1- primer 16; 5'-TCTGCGGAACCGGTGAGTAC-3', JFH1-primer 17; 5'-GCACTCGCAAGCACCCTAT-3', FAM probe; 6-FAMAAAGGCCTTGTGGTACTG-MGB) was used for comparison, whilst the Abbott real-time RT-PCR assay was used as a gold-standard HCV detection reference assay. Each master mix consisted of $2 \times$ TaqMan Fast Universal Mix (Thermo Fisher Scientific), $18 \mu \mathrm{M}$ forward and reverse primers, $5 \mu \mathrm{M}$ probe and $1 \mu \mathrm{L}$ cDNA template in a final volume of $10 \mu \mathrm{L}$. The run consisted of $20 \mathrm{~s}$ hold at $95^{\circ} \mathrm{C}$ followed by 40 cycles of denaturation for $20 \mathrm{~s}$ at $95^{\circ} \mathrm{C}$ and amplification for $30 \mathrm{~s}$ at $60^{\circ} \mathrm{C}$.

Statistical analysis. Normal distribution of data was determined in GraphPad Prism version 7 using D'Agostino \& Person normality test. A parametric one-way ANOVA was used for normally distributed data and a non-parametric KruskalWallis test with Dunn's multiple comparisons test was used for other data types. ROC curves were plotted on the same software. Data were considered significant if $p$ value was less than or equal to 0.05 .

Reporting summary. Further information on research design is available in the Nature Research Reporting Summary linked to this article.

\section{Data availability}

The raw data, sequence alignment, gel blots and images that support the findings of this study are also available in University of Glasgow's Enlighten: Research Data with the identifier: https://doi.org/10.5525/gla.researchdata.1127. Source data are provided with this paper.

Received: 29 March 2021; Accepted: 27 October 2021; Published online: 30 November 2021

\section{References}

1. European Association for the Study of the Liver. EASL Clinical Practice Guidelines: Management of hepatitis C virus infection. J. Hepatol. 60, 392-420 (2014).

2. World Health Organization. Guidelines for the Screening Care and Treatment of Persons with Chronic Hepatitis C Infection: Updated Version (World Health Organization, 2016).

3. Chevaliez, S. \& Pawlotsky, J.-M. Hepatitis $\mathrm{C}$ virus serologic and virologic tests and clinical diagnosis of HCV-related liver disease. Int J. Med Sci. 3, 35-40 (2006).

4. Iversen, J. et al. Estimating the Consensus hepatitis C Cascade of Care among people who inject drugs in Australia: pre and post availability of direct acting antiviral therapy. Int. J. Drug Policy 83, 102837 (2020).

5. Thomson, E. C. et al. Delayed anti-HCV antibody response in HIV-positive men acutely infected with HCV. AIDS 23, 89-93 (2009).

6. Gower, E., Estes, C., Blach, S., Razavi-Shearer, K. \& Razavi, H. Global epidemiology and genotype distribution of the hepatitis $\mathrm{C}$ virus infection. J. Hepatol. 61, S45-S57 (2014).

7. Lamoury, F. M. J. et al. Evaluation of the Xpert HCV viral load finger-stick point-of-care assay. J. Infect. Dis. 217, 1889-1896 (2018).

8. Llibre, A. et al. Development and clinical validation of the Genedrive point-ofcare test for qualitative detection of hepatitis C virus. Gut 67, 2017-2024 (2018)

9. Walker, P. J. et al. Changes to virus taxonomy and the statutes ratified by the international committee on taxonomy of viruses (2020). Arch. Virol. 165, 2737-2748 (2020) 
10. Borgia, S. M. et al. Identification of a novel hepatitis $C$ virus genotype from punjab, india: expanding classification of hepatitis $\mathrm{C}$ virus into 8 genotypes. $J$. Infect. Dis. 218, 1722-1729 (2018).

11. Notomi, T. et al. Loop-mediated isothermal amplification of DNA. Nucleic Acids Res. 28, e63-e63 (2000).

12. Nagamine, K., Hase, T. \& Notomi, T. Accelerated reaction by loop-mediated isothermal amplification using loop primers. Mol. Cell. Probes 16, 223-229 (2002).

13. Viana, G. M. R. et al. Field evaluation of a real time loop-mediated isothermal amplification assay (RealAmp) for malaria diagnosis in Cruzeiro do Sul, Acre, Brazil. PLoS ONE 13, e0200492 (2018).

14. Howson, E. L. A. et al. Evaluation of two lyophilized molecular assays to rapidly detect foot-and-mouth disease virus directly from clinical samples in field settings. Transbound. Emerg. Dis. 64, 861-871 (2017).

15. Reboud, J. et al. Paper-based microfluidics for DNA diagnostics of malaria in low resource underserved rural communities. PNAS 116, 4834-4842 (2019).

16. Hongjaisee, $S$. et al. Rapid visual detection of hepatitis $C$ virus using a reverse transcription loop-mediated isothermal amplification assay. Int. J. Infect. Dis. 102, 440-445 (2021).

17. Yang, J. et al. Detection of hepatitis C virus by an improved loop-mediated isothermal amplification assay. Arch. Virol. 156, 1387-1396 (2011).

18. Kargar, M., Askari, A., Doosti, A. \& Ghorbani-Dalini, S. Loop-mediated isothermal amplification assay for rapid detection of hepatitis $\mathrm{C}$ virus. Indian J. Virol. 23, 18-23 (2012).

19. Wang, Q. et al. Rapid detection of hepatitis C virus RNA by a reverse transcription loop-mediated isothermal amplification assay. FEMS Immunol. Med. Microbiol. 63, 144-147 (2011).

20. Schnell, G. et al. Hepatitis $\mathrm{C}$ virus genetic diversity by geographic region within genotype 1-6 subtypes among patients treated with glecaprevir and pibrentasvir. PLoS ONE 13, e0205186 (2018)

21. Smith, D. B. et al. Expanded classification of hepatitis $C$ virus into 7 genotypes and 67 subtypes: Updated criteria and genotype assignment web resource. Hepatology 59, 318-327 (2014).

22. Davis, C. et al. Highly diverse hepatitis $\mathrm{C}$ strains detected in sub-saharan africa have unknown susceptibility to direct-acting antiviral treatments. Hepatology 69, 1426-1441 (2019).

23. Witteveldt, J. et al. CD81 is dispensable for hepatitis C virus cell-to-cell transmission in hepatoma cells. J. Gen. Virol. 90, 48-58 (2009).

24. Ivanova Reipold, E. et al. Optimising diagnosis of viraemic hepatitis C infection: the development of a target product profile. BMC Infect. Dis. 17, 707 (2017).

25. Nyan, D.-C. \& Swinson, K. L. A method for rapid detection and genotype identification of hepatitis $C$ virus $1-6$ by one-step reverse transcription loopmediated isothermal amplification. Int. J. Infect. Dis. 43, 30-36 (2016).

26. Lee, S. R. et al. Evaluation of a new, rapid test for detecting HCV infection, suitable for use with blood or oral fluid. J. Virol. Methods 172, 27-31 (2011).

27. Busch, M. P. Insights into the epidemiology, natural history and pathogenesis of hepatitis $\mathrm{C}$ virus infection from studies of infected donors and blood product recipients. Transfus. Clin. Biol. 8, 200-206 (2001).

28. Lamb, L. E. et al. Rapid detection of Zika virus in urine samples and infected mosquitos by reverse transcription-loop-mediated isothermal amplification. Sci. Rep. 8, 3803 (2018).

29. Damhorst, G. L. et al. Smartphone-imaged HIV-1 reverse-transcription loopmediated isothermal amplification (RT-LAMP) on a chip from whole blood. Engineering 1, 324-335 (2015)

30. Ihira, M. et al. Direct detection of human herpesvirus 6 DNA in serum by the loop-mediated isothermal amplification method. J. Clin. Virol. 39, 22-26 (2007).

31. Zou, Y. et al. Nucleic acid purification from plants, animals and microbes in under 30 s. PLoS Biol. 15, e2003916 (2017).

32. Kaarj, K., Akarapipad, P. \& Yoon, J.-Y. Simpler, faster, and sensitive Zika virus assay using smartphone detection of loop-mediated Isothermal amplification on paper microfluidic chips. Sci. Rep. 8, 12438 (2018).

33. Linas, B. P., Wong, A. Y., Schackman, B. R., Kim, A. Y. \& Freedberg, K. A. Cost-effective screening for acute hepatitis $\mathrm{C}$ virus infection in HIV-infected men who have sex with men. Clin. Infect. Dis. 55, 279-290 (2012).

34. Martin, N. K. et al. Can antiviral therapy for hepatitis $\mathrm{C}$ reduce the prevalence of HCV among injecting drug user populations? A modeling analysis of its prevention utility. J. Hepatol. 54, 1137-1144 (2011).

35. Nelson, P. K. et al. Global epidemiology of hepatitis B and hepatitis C in people who inject drugs: results of systematic reviews. Lancet 378, 571-583 (2011).
36. Schackman, B. R. et al. Cost-effectiveness of rapid hepatitis $\mathrm{C}$ virus (HCV) testing and simultaneous rapid HCV and HIV testing in substance abuse treatment programs. Addiction 110, 129-143 (2015).

37. Kim, D. D. et al. Cost-effectiveness model for hepatitis C screening and treatment: implications for Egypt and other countries with high prevalence. Glob. Public Health 10, 296-317 (2015).

38. Jiang, X., Loeb, J. C., Manzanas, C., Lednicky, J. A. \& Fan, Z. H. Valve-enabled sample preparation and RNA amplification in a coffee mug for Zika virus detection. Angew. Chem. Int. Ed. 57, 17211-17214 (2018).

39. Domingues, P., Bamford, C. G. G., Boutell, C. \& McLauchlan, J. Inhibition of hepatitis $\mathrm{C}$ virus RNA replication by ISG15 does not require its conjugation to protein substrates by the HERC5 E3 ligase. J. Gen. Virol. 96, 3236-3242 (2015).

40. NEBioCalculator. https://nebiocalculator.neb.com/\#!/ligation.

\section{Acknowledgements}

The authors would like to Dr. Shantimoy Kar and Dr. Xiaoxiang Yan from the James Watt School of Engineering at the University of Glasgow for their inputs on the design of the lateral flow device. This study was funded in part by Engineering and Physical Sciences Research Council, Grant Number: EP/M508056/1, Medical Research Council Grant Numbers: G0801566, G0901213-92157, MC_PC_16045, MC_UU_12014/1, MR/ K013491/1 and the Wellcome Trust, Grant Number: 102789/Z/13/A. We also acknowledge the support of NHS Research Scotland (NRS) Greater Glasgow and Clyde Biorepository.

\section{Author contributions}

E.C.T., J.M.C. and J.R. supervised the project. A.B.S. determined the viral loads and genotypes of the HCV samples by the Abbott RealTime PCR. A.B.S. and R.G. provided and anonymised the samples for the double-blind studies. C.D. and S.R.S. extracted RNA and conducted cDNA synthesis of all samples for the double-blind study. W.M.W performed the LAMP and in-house RT-PCR assays for the double-blind studies. W.W.M., A.G., G.X. and Z.Y. designed the lateral flow devices. W.W.M. and A.G. manufactured and assembled the lateral flow devices. W.W.M., P.J., J.M.C. and J.R. analysed the data. W.W.M. wrote the original manuscript. E.C.T., J.M.C. and J.R. provided revisions and all authors reviewed and edited the manuscript.

\section{Competing interests}

The authors declare no competing interests.

\section{Additional information}

Supplementary information The online version contains supplementary material available at https://doi.org/10.1038/s41467-021-27076-z.

Correspondence and requests for materials should be addressed to Emma C. Thomson or Jonathan M. Cooper.

Peer review information Nature Communications thanks Maria-Nefeli Tsaloglou and the other, anonymous, reviewer(s) for their contribution to the peer review of this work. Peer reviewer reports are available.

Reprints and permission information is available at http://www.nature.com/reprints

Publisher's note Springer Nature remains neutral with regard to jurisdictional claims in published maps and institutional affiliations.

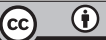

Open Access This article is licensed under a Creative Commons Attribution 4.0 International License, which permits use, sharing, adaptation, distribution and reproduction in any medium or format, as long as you give appropriate credit to the original author(s) and the source, provide a link to the Creative Commons license, and indicate if changes were made. The images or other third party material in this article are included in the article's Creative Commons license, unless indicated otherwise in a credit line to the material. If material is not included in the article's Creative Commons license and your intended use is not permitted by statutory regulation or exceeds the permitted use, you will need to obtain permission directly from the copyright holder. To view a copy of this license, visit http://creativecommons.org/ licenses/by/4.0/.

(C) The Author(s) 2021 